\title{
Extracting and Evaluating Features from RNA Virus Sequences to Predict Host Species Susceptibility Using Deep Learning
}

\author{
Kevin Sutanto \\ kevin.sutanto@uottawa.ca \\ School of Electrical Engineering and Computer Science, \\ University of Ottawa \\ Ottawa, Ontario, Canada
}

\begin{abstract}
Identifying and monitoring hosts of zoonotic RNA viruses, that is, RNA viruses which can be transmitted from one species to another, including the recent SARS-CoV-2 causing the COVID-19 pandemic, is paramount to control their spread. However, efforts to control such spread may be affected if there are unmonitored or unknown hosts. To help identify potential hosts that may harbour such zoonotic viruses, we propose a pipeline that extracts features from sequences of RNA viruses, then uses the extracted features with deep learning to predict host species susceptibility. In addition to using sequence-related features, our method also extracts and uses features derived from the RNA secondary structures that can be formed by the viral sequences, since RNA secondary structures are known to take part in virus-host interaction. We evaluated the performance of our method and the different extracted features with a dataset containing RNA virus sequences and the host they infect, regardless of the viral species, from the NCBI Virus database. Using 10-fold cross validation, we found that a combination of the extracted features yielded the highest overall prediction accuracy of $86.89 \%$.
\end{abstract}

\section{CCS CONCEPTS}

- Applied computing $\rightarrow$ Bioinformatics; Computational biology; • Computing methodologies $\rightarrow$ Machine learning.

\section{KEYWORDS}

RNA virus, host susceptibility, deep learning

\section{ACM Reference Format:}

Kevin Sutanto and Marcel Turcotte. 2021. Extracting and Evaluating Features from RNA Virus Sequences to Predict Host Species Susceptibility Using Deep Learning. In 2021 13th International Conference on Bioinformatics and Biomedical Technology (ICBBT '21), May 21-23, 2021, Xi'an, China. ACM, New York, NY, USA, 9 pages. https://doi.org/10.1145/3473258.3473271

\section{INTRODUCTION}

Monitoring and surveillance of reservoir hosts have been an important strategy to anticipate and control the spread of viruses [28].

*Corresponding author: Marcel Turcotte (marcel.turcottet@uottawa.ca)

\section{(c) (1) (2)}

This work is licensed under a Creative Commons Attribution-Share Alike International 4.0 License.

ICBBT '21, May 21-23, 2021, Xi'an, China

(C) 2021 Copyright held by the owner/author(s)

ACM ISBN 978-1-4503-8965-5/21/05.

https://doi.org/10.1145/3473258.3473271

\author{
Marcel Turcotte* \\ marcel.turcotte@uottawa.ca \\ School of Electrical Engineering and Computer Science, \\ University of Ottawa \\ Ottawa, Ontario, Canada
}

This is especially true for RNA viruses; as they are capable of reassorting themselves during replication, allowing them to "jump" between different host species in a short period of time [44], in addition to their ability to rapidly adapt to novel hosts [7, 8, 26, 43, 46]. Their high mutation rates compared to DNA replication also allows them to have a wider range of susceptible host species [10].

However, such surveillance and control effort may be impacted when there are unknown, unmonitored hosts. This may be the case with the SARS-CoV-2 virus causing the ongoing COVID-19 pandemic, in which researchers believe there may be unknown intermediate hosts between bats and humans [11, 25, 49]. Testing a wide variety of hosts for their susceptibility to the virus, in order to identify these unknown hosts, is demanding. But computational techniques, such as deep neural networks, could be utilized to narrow down the possible unknown hosts, reducing the required tests only to the likely hosts. Deep learning techniques have previously been used to identify viruses from their sequences [39], protein interactions between viral and host proteins [50], and carrier hosts for sequences of "influenza A", "rabies lyssavirus" and "rotavirus A" [31].

Prior work in host identification has mostly relied on the sequences of the viruses themselves [14, 31], the encoded proteins [9], and "word frequency" or k-mers [2, 48]. However, in the case of single-stranded RNA viruses, secondary structure often takes part in their biological and replication processes; and as a result, there may be parts of the viral genomes in which secondary structures are fairly conserved despite the high mutation rate of the nucleotides themselves [17, 41]. For example, alphaviruses were found to have a secondary structure motif whose function is to evade the host viral recognition mechanism [18]. In addition, a recent study has also found conserved structures, "hinting" conserved functions, among the coronaviruses including the recent SARS-CoV-2 [29]. Finally, when it comes to SARS-CoV-2 relative to other viruses in the SARS family, another study has found both structural and nucleotide conservation as well [37]. Due to these roles and conservation of secondary structures in RNA viruses, including their roles in virushost interaction, we decided to investigate if features derived from secondary structures can be used to improve predictions of virushost relationship, in addition to the nucleotide-based features that have been commonly used in previous studies.

Several related host prediction studies focused on certain species of viruses [9, 31], while another focused on the type of host (bacterial hosts) and included hosts and viruses with adequate numbers of sequences [48]. Due to the prevalence of zoonotic RNA viruses [32, 35, 45] (including SARS-CoV-2 [30] and HIV [15]) that can spread from non-human animals to humans, we have decided 
to focus on RNA viruses regardless of the virus species or the host species.

\section{METHODS}

\subsection{Dataset}

Our dataset contains sequences of RNA viruses and their host species. It was obtained from NCBI Virus [3] on September 12, 2020. Only entries that are annotated as being complete (as opposed to partial nucleotides) were included, and duplicate entries were removed. Due to the large memory requirements of matching overly long sequences with our curated RNA secondary structure motifs, we have excluded entries whose viral sequences are longer than 40,000 nucleotides. Furthermore, sequences containing unknown nucleotides and sequences with unknown hosts were also excluded. In addition, as some of the host species have only small numbers of viral sequences in the dataset, we have further filtered the dataset to include only host species with at least 100 viral sequences - similar to how a prior study predicting host genus and virus relationship included only genera with a sufficient number of viral entries, in order to ensure that the learning process has reasonable amount of data points per genus [48].

After all of these filtering steps, we ended up with 47,266 entries (which maximum sequence length is 31,473 instead of our original limit). In total, there are 47 different host species in the included entries. They are as follows: Allium sativum, Anas carolinensis, Anas clypeata, Anas platyrhynchos, Anatidae, Apodemus agrarius, Aves, Bos taurus, Canis lupus familiaris, Capra hircus, Capsicum annuum, Columbidae, Corvus brachyrhynchos, Cricetulus griseus, Culex, Culex pipiens, Culex quinquefasciatus, Culicidae, Culiseta melanura, Cyanocitta cristata, Equus caballus, Felis catus, Gallus gallus, Glycine max, Homo sapiens, Macaca mulatta, Malus domestica, Meleagris gallopavo, Melogale, Mus musculus, Oryza sativa, Ovis aries, Procyon lotor, Prunus, Prunus avium, Prunus persica, Pyrus communis, Rattus norvegicus, Rosa sp., Solanum lycopersicum, Solanum tuberosum, Sus scrofa, Sus scrofa domesticus, Triticum aestivum, Vitis vinifera, Vulpes vulpes, and Zea mays.

\subsection{Secondary Structure Motifs Fingerprinting}

We have previously curated a set of common RNA secondary structure motifs, in order to form secondary structure "fingerprints" to be used as deep learning features [42]. The steps to produce the secondary structure "fingerprints" are as follows: finding matches of the curated RNA secondary structure motifs within the sequence in question; getting free energy values of the matches; substituting non-matches with the maximum free energy value (representing a highly unstable or weak structure); rescaling the values so that maximum free energy for a motif is represented by 0 (poorest or no match), while the minimum free energy for a motif is represented by 1 (structurally most stable match), and the free energy values in between is represented by values between 0 and 1 ; and finally, concatenating the rescaled values together, forming the fingerprint [42]. These steps are illustrated by Figure 1. The secondary structure matching and free energy calculations were performed using the RNAMotif program (the built-in "efn2" method was used to calculate the free energy of matches) [27].

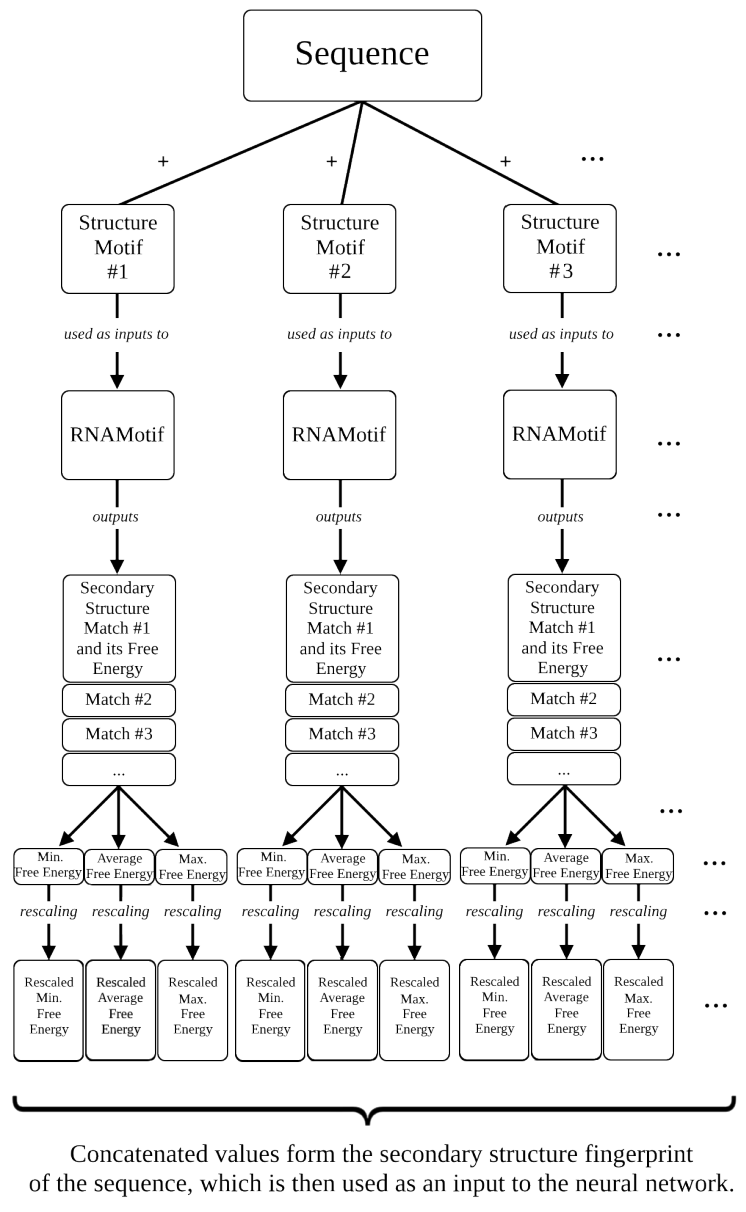

Figure 1: Steps to produce secondary structure fingerprints, using our curation of known RNA secondary structure motifs [42].

In our prior work, for a single secondary structure motif and a single sequence, only the minimum free energy was used (representing the most thermodynamically stable match, if there are multiple matches) [42]. However, for this study, we decided to also include the average free energy (of all matches in single a sequence) and maximum free energy (least stable match if there are multiple match) values as additional features; and investigate if combining them with the minimum free energy values would improve accuracy, compared to using only the minimum free energy by itself.

\subsection{K-mers}

K-mer representations can be considered "signatures" of the corresponding sequences [38]. Using k-mers allows us to infer nucleotide similarities in different sequences, even if the sequences are not 
aligned [20]. They are widely used in bioinformatics, and their usefulness as representations of viral sequences has been observed in previous related studies $[2,48]$.

In this study, k-mers of $k=4,5$, and 6 were used. For a single sequence and a $k$, the k-mers are formed by taking the occurrence counts of each possible nucleotide combination (of length $k$ ) divided by the total of all of the aforementioned occurrences. Therefore, the k-mer distribution using a specific $k$ for a sequence adds up to 1 [42]. Similar to our previous approach of using k-mers as features for deep learning, k-mers were both used alone and in conjunction with other types of features to compare the differences in results.

\subsection{Skip-mers}

Using k-mer representations with larger values of $k$ in order to capture distributions of longer subsequences is CPU and memory intensive - for a length of $k$ with 4 possible nucleotides at each position, a k-mer representation of a sequence will contain $4^{k}$ values. In other words, simply increasing the value of $k$ will result in exponential increase in the dimension of the feature vectors and the memory requirements to train the neural network.

Therefore, in order to capture longer patterns that may be present in the dataset while still keeping the memory requirements reasonable, we have used "skip-mers" [6] as additional features. "Skipmers" [6] are based on the "gapped q-grams" concept [4], in which matches are made with non-consecutive strings. The sparsity of the matching strings can be defined by the number of consecutive characters that have to match (matching characters), and the number of consecutive characters that do not have to match (skip characters) following the matching characters. For the purposes of describing the "skip-mers" [6] used in our study, we define the total number of matching and skip characters to be the length of the "skip-mer" [6]. Therefore, its length would be equal to the length of the strings that fit its criteria. All of the "skip-mers" [6] we used start with matching characters. Matching characters are followed by skip characters, while the latter is followed by the former. In other words, a "skipmer" [6] alternates between consecutive matching characters and consecutive skip characters until the total number of characters matches its required length. A "skip-mer" [6] representation of a single sequence consists of the distribution of all possible combinations of the matching characters (in their corresponding positions) - that is, $4^{m}$ values, where $m$ is the total number of matching characters, in which each matching character can be one of the four possible nucleotides.

We used a set of "skip-mers" [6] with 1 consecutive matching character followed by 1 consecutive skip character, and another set with 2 consecutive matching characters followed by 1 consecutive skip character. The rationale behind the latter set is to capture any possible biases on the first two nucleotides of codons (based on the fact that the third nucleotide is often redundant in amino acid translation [16]), which, if present and not undermined by false matches from non-coding regions or matches with incorrect open reading frames [33], may be useful for the neural network to learn and use as additional features.

Each of the sets contains "skip-mers" [6] with different lengths such that the total number of matching characters are 4,5 , and 6 (which are the same as the numbers of characters that have to match in the k-mer features). To summarize, the "skip-mers" [6] we used are as follows:

- Match 1 Skip 1 with a length of 7 (4 matching characters in total),

- Match 1 Skip 1 with a length of 9 (5 matching characters in total),

- Match 1 Skip 1 with a length of 11 (6 matching characters in total),

- Match 2 Skip 1 with a length of 6 (4 matching characters in total),

- Match 2 Skip 1 with a length of 7 (5 matching characters in total), and

- Match 2 Skip 1 with a length of 9 (6 matching characters in total).

Similar to the two other types of features, the "skip-mers" [6] are both used alone and combined with the other feature types. When it comes to using them along with k-mers, those with the same number of matching characters were paired together. For example, $\mathrm{k}$-mer with $k=4$ was used together with the match 1 skip 1 "skip-mer" [6] of length 7.

For each of the above configurations of "skip-mer" [6] and for a single sequence, similar to our approach with k-mers, the number of matches with each of the possible strings is counted. Each of these counts is then divided by the sum of the counts, forming a probability distribution of matches with the possible strings within the sequence. This distribution of matches is then used as the neural network input vector representing the sequence.

\subsection{Deep Learning Models}

Deep learning was used in a related study on host prediction from sequences of specific viral species [31], in which the authors had found that their deep learning based approach outperformed a preceding study of similar datasets using a different approach [21] [31]. Other studies involving RNA or DNA sequences have also found deep learning based approaches to be dependable [23], likely due to the ability of deep neural networks to recognize and uncover subtle patterns [40].

For each set of features (of a single type or multiple/combined types, as previously discussed), 3 deep neural network architectures differing only in depths were used, as we found that certain types or combination of features worked better with shallow networks while others worked better with deeper networks. The size of the input vector and the width of the network depend on the feature set being used. For sets consisting only of a single feature type, the input vector length and the network width are equal to the number of values representing an input sequence (e.g. $4^{k}$ for a feature set consisting only of k-mer for a specific $k$ ). Meanwhile, for sets with more than one feature type in it, the input vector length and network width are equal to the total number of values from all of the included features, as the values from the different features are concatenated. In all of the network architectures; each layer, except the last/output layer, is fully connected to the next layer (i.e. densely connected).

The 3 different neural network architectures are as follows:

- 2 consecutive dense layers with the "relu" activation function [34] (each of the layers is $n$ in width, where $n$ is the 
number of values in the feature set being used), followed by a final dense layer with the "softmax" activation function (total depth = 3);

- 3 consecutive "relu"-activated [34] dense layers (each is $n$ in width), followed by a "softmax"-activated dense layer (total depth =4); and

- 4 consecutive "relu"-activated [34] dense layers (each is $n$ in width), followed by a "softmax"-activated dense layer (total depth $=5$ ).

The best of the 3 results from the different network architectures is then considered as the performance of the feature set. These architectures and other configurations, such as the number of epochs and learning rates, were determined by trying out different configurations, initially with early stopping estimating the optimal number of epochs and learning rates. This was done using $80 \%$ of the dataset as training set, $10 \%$ as validation set (for early stopping), and the last $10 \%$ as final test set. However, the training and evaluation of the final models were performed without early stopping, with $90 \%$ of entries used as training set, and the remaining $10 \%$ used as test set. These final models used to evaluate the performance of each feature set were trained using the "Adam" optimizer [19] for 300 epochs, starting with the default learning rate of 0.001 for the first 100 epochs, then reduced by half for each of the next 100 epochs (i.e. 0.0005 for epochs 101-200, then 0.00025 for epochs 201300). Keras [5] with the Tensorflow backend [1] was used to build, train, and evaluate the deep neural networks. The sparse categorical crossentropy function built into Keras [1] was used as the loss function.

\section{RESULTS AND DISCUSSION}

Similar to our previous study on RNA secondary structure fingerprints [42], we evaluate the performance of the classifier trained using the different feature sets with 10 -fold cross validation [13]. This means that the training and evaluation processes for each feature set were performed 10 times, in which the training and evaluation sets in each repetition differed from the others. In addition, as per our previous approach [42], the data splitting into different folds takes the distribution of hosts into account, which means that each fold has the same distribution of host species in it.

\subsection{Primary Evaluation}

Table 1 shows the features used in each set, and their 10 -fold cross validation overall accuracy followed by the standard errors. The accuracy reported takes class imbalances into account. For each set, the highest accuracy achieved is indicated in bold.

When using only features of a single type at a time, sequencebased features (i.e. k-mers and "skip-mers" [6]) outperformed secondary structure based features in this study, with the longest "skip-mer" [6] matching 2 nucleotides then skipping 1 nucleotide performed best. When it comes to the secondary structure fingerprints, it's also noticeable that combining the different scores derived from the minimum free energy (representing the most thermodynamically stable match of the secondary structure in the sequence), average free energy, and maximum free energy (least thermodynamically stable) of matches produced better results than using only the scores derived from the minimum free energy alone.
The highest accuracy at $86.89 \%$ was obtained by combining 6-mer with the match-2-skip-1 "skip-mer" [6] of length 9. None of the combined features of all 3 types managed to exceed this accuracy rate.

\subsection{Additional Training and Evaluation with Limited Dataset}

In order to test if the best performing feature set is consistent in datasets of different sizes, we have also evaluated our pipeline when trained with a limited amount of data. To perform this additional evaluation, we produced 2 different limited datasets: one is produced by keeping only sequences that are at most $80 \%$ similar using the CD-hit tool $[12,22]$, while the other only has sequences that are at most $90 \%$ similar. The lowest similarity threshold we used has been chosen by other studies involving RNA sequences as well [24, 36, $42,47]$. We used the same set of hosts/classes with our $80 \%$ and $90 \%$ datasets, but different than the set of hosts used with our primary dataset. This is due to the limited number of samples per class in these limited datasets. In addition, we reduced the minimum number of entries per class from 100 to 50 , counted on our $80 \%$ dataset. In other words, only hosts that have at least 50 entries in the $80 \%$ dataset are used. We ended up with a set of 14 hosts, and they are as follows: Allium sativum, Arthropoda, Astacoidea, Bos taurus, Culicidae, Gallus gallus, Hexapoda, Homo sapiens, Octopus, Odonata, Sarcosphaera coronaria, Solanum lycopersicum, Sus scrofa, and Vitis vinifera. In the $80 \%$ dataset, 3,163 entries are of those hosts and therefore used; while in the $90 \%$ dataset, 5,781 entries are used.

Unlike the primary dataset evaluation, 10 -fold cross validation was not used in this preliminary evaluation with the limited datasets (the standard errors of the 10 -fold cross validation in the primary evaluation were found to be small as well). For each of the training and evaluation processes, $80 \%$ of the entries were used as training set, $10 \%$ used as validation set (for early stopping), and another $10 \%$ used as test set (to evaluate the final accuracy). Other than the aforementioned differences, the architectures of the deep learning models are the same as the models we used for the primary evaluation.

The resulting evaluation accuracy of each feature set for the $80 \%$ dataset is listed in table 2, while table 3 lists the results for the $90 \%$ dataset. Our findings indicate that the best performing set of features differed between each of the datasets. 5-mer combined with the match-2-skip-1 "skip-mer" of length 7 [6] performed best with the $80 \%$ dataset at $60.54 \%$ test set accuracy, while 6-mer alone performed best with the $90 \%$ dataset at $80.51 \%$. These suggest that the best performing set of features on our primary evaluation may still not be the best when used with a more complete, future dataset.

\section{CONCLUSIONS}

To help control the spread of zoonotic RNA viruses, we proposed and tested a deep learning based pipeline to predict susceptible hosts (which may act as reservoirs for the viruses) from the viral sequence. Different types of features were extracted from the viral sequences, and their performance as inputs to the deep neural network were evaluated. These different types of features evaluated in this study are k-mers, "skip-mers" [6], and secondary structure fingerprints [42]. Unlike prior studies in host predictions [9, 21, 31], 
Table 1: 10-fold cross validation results (accuracy and standard errors) for each feature set

\begin{tabular}{|c|c|c|c|c|c|c|c|}
\hline \multirow[t]{2}{*}{ K-mer } & \multicolumn{3}{|c|}{ "Skip-mer” [6] } & \multirow[t]{2}{*}{ Secondary Structure Fingerprints } & \multicolumn{3}{|c|}{ 10-Fold Cross Validation Averaged Accuracy } \\
\hline & Length & Match & Skip & & 3-Layers Model & 4-Layers Model & 5-Layers Model \\
\hline 4 -mer & & - & & - & $62.48 \% \pm 0.51 \%$ & $64.86 \% \pm 0.76 \%$ & $62.09 \% \pm 0.77 \%$ \\
\hline 5-mer & & - & & - & $77.29 \% \pm 0.22 \%$ & $75.24 \% \pm 0.53 \%$ & $74.31 \% \pm 0.46 \%$ \\
\hline 6-mer & & - & & - & $\mathbf{8 4 . 5 6 \%} \pm 0.28 \%$ & $83.55 \% \pm 0.48 \%$ & $83.55 \% \pm 0.57 \%$ \\
\hline- & 6 & 2 & 1 & - & $61.74 \% \pm 0.31 \%$ & $61.85 \% \pm 0.94 \%$ & $59.45 \% \pm 1.0 \%$ \\
\hline- & 7 & 1 & 1 & - & $\mathbf{5 5 . 8 9 \%} \pm 0.34 \%$ & $54.38 \% \pm 0.99 \%$ & $48.39 \% \pm 1.86 \%$ \\
\hline- & 7 & 2 & 1 & - & $77.32 \% \pm 0.5 \%$ & $75.76 \% \pm 0.8 \%$ & $71.74 \% \pm 1.83 \%$ \\
\hline- & 9 & 1 & 1 & - & $75.16 \% \pm 0.41 \%$ & $73.23 \% \pm 0.46 \%$ & $65.53 \% \pm 4.57 \%$ \\
\hline- & 9 & 2 & 1 & - & $84.92 \% \pm 0.25 \%$ & $84.0 \% \pm 0.36 \%$ & $82.2 \% \pm 1.13 \%$ \\
\hline- & 11 & 1 & 1 & - & $\mathbf{8 4 . 0 8 \%} \pm 0.21 \%$ & $81.78 \% \pm 0.98 \%$ & $81.15 \% \pm 0.88 \%$ \\
\hline- & & - & & $\min$. free energy & $35.91 \% \pm 0.42 \%$ & $36.75 \% \pm 0.76 \%$ & $35.94 \% \pm 0.51 \%$ \\
\hline- & & - & & min., avg. free energy & $50.65 \% \pm 0.57 \%$ & $52.04 \% \pm 0.86 \%$ & $\mathbf{5 2 . 6 \%} \pm 0.65 \%$ \\
\hline- & & - & & min., avg., max. free energy & $57.37 \% \pm 0.52 \%$ & $59.39 \% \pm 0.58 \%$ & $\mathbf{5 9 . 4 2} \% \pm 0.76 \%$ \\
\hline 4 -mer & 6 & 2 & 1 & - & $71.57 \% \pm 0.4 \%$ & $71.69 \% \pm 0.41 \%$ & $71.15 \% \pm 0.49 \%$ \\
\hline 4 -mer & 7 & 1 & 1 & - & $70.52 \% \pm 0.39 \%$ & $71.91 \% \pm 0.38 \%$ & $69.63 \% \pm 1.01 \%$ \\
\hline 5 -mer & 7 & 2 & 1 & - & $\mathbf{8 2 . 1 4 \%} \pm 0.29 \%$ & $82.08 \% \pm 0.46 \%$ & $80.1 \% \pm 0.65 \%$ \\
\hline 5-mer & 9 & 1 & 1 & - & $\mathbf{8 1 . 7 7 \%} \pm 0.47 \%$ & $81.13 \% \pm 0.39 \%$ & $80.39 \% \pm 0.69 \%$ \\
\hline 6-mer & 9 & 2 & 1 & - & $\mathbf{8 6 . 8 9 \%} \pm 0.28 \%$ & $86.09 \% \pm 0.21 \%$ & $84.68 \% \pm 0.83 \%$ \\
\hline 6-mer & 11 & 1 & 1 & - & $86.7 \% \pm 0.38 \%$ & $86.17 \% \pm 0.61 \%$ & $84.73 \% \pm 1.58 \%$ \\
\hline 4-mer & & - & & $\min$. free energy & $67.96 \% \pm 0.56 \%$ & $70.97 \% \pm 0.54 \%$ & $72.6 \% \pm 0.63 \%$ \\
\hline 5-mer & & - & & $\min$. free energy & $78.93 \% \pm 0.24 \%$ & $80.49 \% \pm 0.62 \%$ & $81.05 \% \pm 0.46 \%$ \\
\hline 6-mer & & - & & $\min$. free energy & $84.33 \% \pm 0.51 \%$ & $84.05 \% \pm 0.71 \%$ & $77.7 \% \pm 5.36 \%$ \\
\hline 4 -mer & & - & & min., avg. free energy & $69.92 \% \pm 0.56 \%$ & $72.28 \% \pm 0.52 \%$ & $75.38 \% \pm 0.6 \%$ \\
\hline 5-mer & & - & & min., avg. free energy & $74.93 \% \pm 1.66 \%$ & $\mathbf{8 1 . 2 8 \%} \pm 0.43 \%$ & $81.02 \% \pm 0.33 \%$ \\
\hline 6-mer & & - & & min., avg. free energy & $83.42 \% \pm 0.39 \%$ & $83.73 \% \pm 0.32 \%$ & $82.23 \% \pm 0.32 \%$ \\
\hline 4 -mer & & - & & min., avg., max. free energy & $71.14 \% \pm 0.49 \%$ & $74.63 \% \pm 0.54 \%$ & $75.85 \% \pm 0.54 \%$ \\
\hline 5-mer & & - & & min., avg., max. free energy & $79.28 \% \pm 0.75 \%$ & $80.74 \% \pm 0.52 \%$ & $\mathbf{8 1 . 2 3 \%} \pm 0.75 \%$ \\
\hline 6-mer & & - & & min., avg., max. free energy & $83.21 \% \pm 0.37 \%$ & $83.53 \% \pm 0.13 \%$ & $81.87 \% \pm 0.44 \%$ \\
\hline- & 6 & 2 & 1 & min. free energy & $66.94 \% \pm 0.58 \%$ & $69.98 \% \pm 0.65 \%$ & $71.02 \% \pm 0.83 \%$ \\
\hline- & 7 & 1 & 1 & $\min$. free energy & $66.83 \% \pm 0.22 \%$ & $69.69 \% \pm 0.48 \%$ & $71.23 \% \pm 0.34 \%$ \\
\hline- & 7 & 2 & 1 & $\min$. free energy & $78.66 \% \pm 0.55 \%$ & $80.35 \% \pm 0.41 \%$ & $\mathbf{8 0 . 7 2 \%} \pm 0.59 \%$ \\
\hline- & 9 & 1 & 1 & $\min$. free energy & $77.78 \% \pm 0.27 \%$ & $\mathbf{8 0 . 0 5 \%} \pm 0.29 \%$ & $79.43 \% \pm 1.73 \%$ \\
\hline- & 9 & 2 & 1 & $\min$. free energy & $\mathbf{8 4 . 5 8 \%} \pm 0.34 \%$ & $79.17 \% \pm 3.18 \%$ & $80.65 \% \pm 1.28 \%$ \\
\hline- & 11 & 1 & 1 & $\min$. free energy & $83.61 \% \pm 0.52 \%$ & $\mathbf{8 3 . 8 8 \%} \pm 0.32 \%$ & $77.77 \% \pm 5.17 \%$ \\
\hline- & 6 & 2 & 1 & min., avg. free energy & $69.62 \% \pm 0.49 \%$ & $71.83 \% \pm 0.74 \%$ & $74.16 \% \pm 0.6 \%$ \\
\hline- & 7 & 1 & 1 & min., avg. free energy & $68.11 \% \pm 0.99 \%$ & $71.45 \% \pm 0.73 \%$ & $73.93 \% \pm 0.59 \%$ \\
\hline- & 7 & 2 & 1 & min., avg. free energy & $78.64 \% \pm 0.35 \%$ & $79.75 \% \pm 0.85 \%$ & $\mathbf{8 0 . 9 \%} \pm 0.32 \%$ \\
\hline- & 9 & 1 & 1 & min., avg. free energy & $78.48 \% \pm 0.59 \%$ & $79.58 \% \pm 0.55 \%$ & $\mathbf{8 1 . 2 9 \%} \pm 0.32 \%$ \\
\hline- & 9 & 2 & 1 & min., avg. free energy & $83.2 \% \pm 0.54 \%$ & $83.37 \% \pm 0.43 \%$ & $82.45 \% \pm 0.57 \%$ \\
\hline- & 11 & 1 & 1 & min., avg. free energy & $\mathbf{8 2 . 8 3 \%} \pm 0.41 \%$ & $82.75 \% \pm 0.44 \%$ & $82.3 \% \pm 0.45 \%$ \\
\hline- & 6 & 2 & 1 & min., avg., max. free energy & $70.77 \% \pm 0.42 \%$ & $74.04 \% \pm 0.75 \%$ & $75.38 \% \pm 0.36 \%$ \\
\hline- & 7 & 1 & 1 & min., avg., max. free energy & $69.28 \% \pm 0.75 \%$ & $74.32 \% \pm 0.52 \%$ & $\mathbf{7 4 . 7 4 \%} \pm 0.75 \%$ \\
\hline- & 7 & 2 & 1 & min., avg., max. free energy & $79.02 \% \pm 0.58 \%$ & $80.43 \% \pm 0.52 \%$ & $\mathbf{8 1 . 1 6 \%} \pm 0.52 \%$ \\
\hline- & 9 & 1 & 1 & min., avg., max. free energy & $78.73 \% \pm 0.45 \%$ & $80.42 \% \pm 0.7 \%$ & $\mathbf{8 1 . 3 \%} \pm 0.33 \%$ \\
\hline- & 9 & 2 & 1 & min., avg., max. free energy & $83.93 \% \pm 0.2 \%$ & $83.38 \% \pm 0.42 \%$ & $82.34 \% \pm 0.63 \%$ \\
\hline- & 11 & 1 & 1 & min., avg., max. free energy & $\mathbf{8 3 . 0 4 \%} \pm 0.4 \%$ & $83.0 \% \pm 0.18 \%$ & $82.47 \% \pm 0.35 \%$ \\
\hline 4 -mer & 6 & 2 & 1 & $\min$. free energy & $74.26 \% \pm 0.46 \%$ & $76.11 \% \pm 0.66 \%$ & $78.78 \% \pm 0.3 \%$ \\
\hline 4 -mer & 7 & 1 & 1 & min. free energy & $74.22 \% \pm 0.29 \%$ & $77.44 \% \pm 0.83 \%$ & $\mathbf{7 8 . 5 4 \%} \pm 0.47 \%$ \\
\hline 5-mer & 7 & 2 & 1 & $\min$. free energy & $\mathbf{8 3 . 7 4 \%} \pm 0.39 \%$ & $83.54 \% \pm 0.39 \%$ & $83.65 \% \pm 0.18 \%$ \\
\hline 5-mer & 9 & 1 & 1 & $\min$. free energy & $82.17 \% \pm 0.47 \%$ & $83.21 \% \pm 0.42 \%$ & $83.07 \% \pm 0.45 \%$ \\
\hline 6-mer & 9 & 2 & 1 & $\min$. free energy & $\mathbf{8 5 . 9 \%} \pm 0.28 \%$ & $84.37 \% \pm 0.71 \%$ & $83.1 \% \pm 0.73 \%$ \\
\hline 6-mer & 11 & 1 & 1 & $\min$. free energy & $\mathbf{8 5 . 8 6 \%} \pm 0.35 \%$ & $84.94 \% \pm 0.34 \%$ & $82.39 \% \pm 0.57 \%$ \\
\hline 4 -mer & 6 & 2 & 1 & min., avg. free energy & $75.06 \% \pm 0.53 \%$ & $77.33 \% \pm 0.66 \%$ & $78.89 \% \pm 0.48 \%$ \\
\hline 4 -mer & 7 & 1 & 1 & min., avg. free energy & $74.78 \% \pm 0.46 \%$ & $77.11 \% \pm 0.39 \%$ & $78.47 \% \pm 0.48 \%$ \\
\hline 5-mer & 7 & 2 & 1 & min., avg. free energy & $82.52 \% \pm 0.38 \%$ & $82.77 \% \pm 0.41 \%$ & $82.68 \% \pm 0.27 \%$ \\
\hline 5 -mer & 9 & 1 & 1 & min., avg. free energy & $81.26 \% \pm 0.38 \%$ & $82.59 \% \pm 0.6 \%$ & $82.37 \% \pm 0.41 \%$ \\
\hline 6-mer & 9 & 2 & 1 & min., avg. free energy & $84.39 \% \pm 0.52 \%$ & $84.2 \% \pm 0.3 \%$ & $82.54 \% \pm 1.01 \%$ \\
\hline 6-mer & 11 & 1 & 1 & min., avg. free energy & $84.33 \% \pm 0.53 \%$ & $84.19 \% \pm 0.65 \%$ & $82.06 \% \pm 0.7 \%$ \\
\hline 4 -mer & 6 & 2 & 1 & min., avg., max. free energy & $75.73 \% \pm 0.57 \%$ & $79.56 \% \pm 0.2 \%$ & $79.65 \% \pm 0.67 \%$ \\
\hline 4 -mer & 7 & 1 & 1 & min., avg., max. free energy & $75.77 \% \pm 0.61 \%$ & $77.99 \% \pm 0.38 \%$ & $79.23 \% \pm 0.44 \%$ \\
\hline 5 -mer & 7 & 2 & 1 & min., avg., max. free energy & $82.54 \% \pm 0.39 \%$ & $\mathbf{8 2 . 9 2 \%} \pm 0.28 \%$ & $82.16 \% \pm 0.61 \%$ \\
\hline 5 -mer & 9 & 1 & 1 & min., avg., max. free energy & $81.41 \% \pm 0.34 \%$ & $\mathbf{8 3 . 1 3 \%} \pm 0.17 \%$ & $81.55 \% \pm 1.31 \%$ \\
\hline 6-mer & 9 & 2 & 1 & min., avg., max. free energy & $84.73 \% \pm 0.32 \%$ & $83.71 \% \pm 0.16 \%$ & $82.33 \% \pm 0.64 \%$ \\
\hline 6-mer & 11 & 1 & 1 & min., avg., max. free energy & $84.61 \% \pm 0.26 \%$ & $83.33 \% \pm 0.68 \%$ & $80.4 \% \pm 2.18 \%$ \\
\hline
\end{tabular}


Table 2: Performance of each feature set when trained and evaluated with a limited dataset without sequences that are more than $80 \%$ similar

\begin{tabular}{|c|c|c|c|c|c|c|c|}
\hline \multirow[t]{2}{*}{ K-mer } & \multicolumn{3}{|c|}{ Skip-mer } & \multirow[t]{2}{*}{ Secondary Structure Fingerprints } & \multicolumn{3}{|c|}{ Test Set Accuracy } \\
\hline & Length & Match & Skip & & 3-Layers Model & 4-Layers Model & 5-Layers Model \\
\hline 4 -mer & & - & & - & $44.22 \%$ & $41.5 \%$ & $40.14 \%$ \\
\hline 5-mer & & - & & - & $46.26 \%$ & $48.3 \%$ & $35.37 \%$ \\
\hline 6-mer & & - & & - & $53.74 \%$ & $47.62 \%$ & $36.73 \%$ \\
\hline- & 6 & 2 & 1 & - & $31.97 \%$ & $46.26 \%$ & $42.86 \%$ \\
\hline- & 7 & 1 & 1 & - & $33.33 \%$ & $36.73 \%$ & $35.37 \%$ \\
\hline- & 7 & 2 & 1 & - & $54.42 \%$ & $34.01 \%$ & $36.73 \%$ \\
\hline- & 9 & 1 & 1 & - & $42.18 \%$ & $35.37 \%$ & $32.65 \%$ \\
\hline- & 9 & 2 & 1 & - & $46.26 \%$ & $49.66 \%$ & $19.05 \%$ \\
\hline- & 11 & 1 & 1 & - & $47.62 \%$ & $26.53 \%$ & $26.53 \%$ \\
\hline- & & - & & $\min$. free energy & $25.17 \%$ & $25.17 \%$ & $31.29 \%$ \\
\hline- & & - & & min., avg. free energy & $31.29 \%$ & $31.97 \%$ & $32.65 \%$ \\
\hline- & & - & & min., avg., max. free energy & $35.37 \%$ & $36.05 \%$ & $33.33 \%$ \\
\hline 4 -mer & 6 & 2 & 1 & - & $45.58 \%$ & $48.3 \%$ & $50.34 \%$ \\
\hline 4 -mer & 7 & 1 & 1 & - & $44.9 \%$ & $44.22 \%$ & $43.54 \%$ \\
\hline 5 -mer & 7 & 2 & 1 & - & $60.54 \%$ & $56.46 \%$ & $39.46 \%$ \\
\hline 5 -mer & 9 & 1 & 1 & - & $59.18 \%$ & $50.34 \%$ & $50.34 \%$ \\
\hline 6-mer & 9 & 2 & 1 & - & $\mathbf{5 7 . 1 4 \%}$ & $50.34 \%$ & $42.18 \%$ \\
\hline 6-mer & 11 & 1 & 1 & - & $57.82 \%$ & $44.9 \%$ & $42.86 \%$ \\
\hline 4 -mer & & - & & $\min$. free energy & $30.61 \%$ & $31.29 \%$ & $41.5 \%$ \\
\hline 5 -mer & & - & & $\min$. free energy & $44.22 \%$ & $46.94 \%$ & $35.37 \%$ \\
\hline 6-mer & & - & & $\min$. free energy & $43.54 \%$ & $44.22 \%$ & $42.86 \%$ \\
\hline 4 -mer & & - & & min., avg. free energy & $37.41 \%$ & $42.86 \%$ & $40.82 \%$ \\
\hline 5-mer & & - & & min., avg. free energy & $49.66 \%$ & $48.3 \%$ & $42.86 \%$ \\
\hline 6-mer & & - & & min., avg. free energy & $40.14 \%$ & $46.26 \%$ & $44.22 \%$ \\
\hline 4 -mer & & - & & min., avg., max. free energy & $44.9 \%$ & $37.41 \%$ & $35.37 \%$ \\
\hline 5 -mer & & - & & min., avg., max. free energy & $42.86 \%$ & $36.73 \%$ & $36.05 \%$ \\
\hline 6-mer & & - & & min., avg., max. free energy & $49.66 \%$ & $42.18 \%$ & $27.89 \%$ \\
\hline- & 6 & 2 & 1 & min. free energy & $39.46 \%$ & $35.37 \%$ & $30.61 \%$ \\
\hline- & 7 & 1 & 1 & $\min$. free energy & $36.73 \%$ & $36.73 \%$ & $37.41 \%$ \\
\hline- & 7 & 2 & 1 & $\min$. free energy & $46.94 \%$ & $51.02 \%$ & $36.73 \%$ \\
\hline- & 9 & 1 & 1 & $\min$. free energy & $38.1 \%$ & $34.69 \%$ & $33.33 \%$ \\
\hline- & 9 & 2 & 1 & $\min$. free energy & $51.02 \%$ & $48.98 \%$ & $37.41 \%$ \\
\hline- & 11 & 1 & 1 & $\min$. free energy & $34.69 \%$ & $34.69 \%$ & $25.85 \%$ \\
\hline- & 6 & 2 & 1 & min., avg. free energy & $36.05 \%$ & $33.33 \%$ & $31.97 \%$ \\
\hline- & 7 & 1 & 1 & min., avg. free energy & $32.65 \%$ & $31.97 \%$ & $34.01 \%$ \\
\hline- & 7 & 2 & 1 & min., avg. free energy & $38.78 \%$ & $44.9 \%$ & $44.9 \%$ \\
\hline- & 9 & 1 & 1 & min., avg. free energy & $36.73 \%$ & $34.01 \%$ & $34.69 \%$ \\
\hline- & 9 & 2 & 1 & min., avg. free energy & $42.86 \%$ & $37.41 \%$ & $36.73 \%$ \\
\hline- & 11 & 1 & 1 & min., avg. free energy & $34.69 \%$ & $31.97 \%$ & $33.33 \%$ \\
\hline- & 6 & 2 & 1 & min., avg., max. free energy & $27.89 \%$ & $34.01 \%$ & $43.54 \%$ \\
\hline- & 7 & 1 & 1 & min., avg., max. free energy & $36.05 \%$ & $29.93 \%$ & $43.54 \%$ \\
\hline- & 7 & 2 & 1 & min., avg., max. free energy & $40.82 \%$ & $38.1 \%$ & $35.37 \%$ \\
\hline- & 9 & 1 & 1 & min., avg., max. free energy & $38.78 \%$ & $38.78 \%$ & $42.18 \%$ \\
\hline- & 9 & 2 & 1 & min., avg., max. free energy & $44.9 \%$ & $43.54 \%$ & $39.46 \%$ \\
\hline- & 11 & 1 & 1 & min., avg., max. free energy & $43.54 \%$ & $38.1 \%$ & $35.37 \%$ \\
\hline 4 -mer & 6 & 2 & 1 & min. free energy & $46.94 \%$ & $45.58 \%$ & $40.82 \%$ \\
\hline 4 -mer & 7 & 1 & 1 & $\min$. free energy & $35.37 \%$ & $41.5 \%$ & $29.93 \%$ \\
\hline 5-mer & 7 & 2 & 1 & $\min$. free energy & $48.98 \%$ & $46.94 \%$ & $45.58 \%$ \\
\hline 5 -mer & 9 & 1 & 1 & $\min$. free energy & $54.42 \%$ & $48.98 \%$ & $37.41 \%$ \\
\hline 6-mer & 9 & 2 & 1 & $\min$. free energy & $36.73 \%$ & $42.86 \%$ & $44.22 \%$ \\
\hline 6-mer & 11 & 1 & 1 & $\min$. free energy & $53.74 \%$ & $42.86 \%$ & $36.05 \%$ \\
\hline 4 -mer & 6 & 2 & 1 & min., avg. free energy & $37.41 \%$ & $43.54 \%$ & $38.78 \%$ \\
\hline 4 -mer & 7 & 1 & 1 & min., avg. free energy & $47.62 \%$ & $44.22 \%$ & $44.9 \%$ \\
\hline 5-mer & 7 & 2 & 1 & min., avg. free energy & $44.9 \%$ & $47.62 \%$ & $37.41 \%$ \\
\hline 5-mer & 9 & 1 & 1 & min., avg. free energy & $40.82 \%$ & $40.14 \%$ & $46.26 \%$ \\
\hline 6-mer & 9 & 2 & 1 & min., avg. free energy & $53.06 \%$ & $32.65 \%$ & $35.37 \%$ \\
\hline 6-mer & 11 & 1 & 1 & min., avg. free energy & $40.14 \%$ & $40.14 \%$ & $36.05 \%$ \\
\hline 4 -mer & 6 & 2 & 1 & min., avg., max. free energy & $46.94 \%$ & $45.58 \%$ & $42.86 \%$ \\
\hline 4 -mer & 7 & 1 & 1 & min., avg., max. free energy & $39.46 \%$ & $45.58 \%$ & $42.18 \%$ \\
\hline 5 -mer & 7 & 2 & 1 & min., avg., max. free energy & $44.22 \%$ & $50.34 \%$ & $47.62 \%$ \\
\hline 5 -mer & 9 & 1 & 1 & min., avg., max. free energy & $48.98 \%$ & $48.3 \%$ & $42.86 \%$ \\
\hline 6-mer & 9 & 2 & 1 & min., avg., max. free energy & $45.58 \%$ & $42.18 \%$ & $32.65 \%$ \\
\hline 6-mer & 11 & 1 & 1 & min., avg., max. free energy & $50.34 \%$ & $46.26 \%$ & $40.14 \%$ \\
\hline
\end{tabular}


Table 3: Performance of each feature set when trained and evaluated with a limited dataset without sequences that are more than $90 \%$ similar

\begin{tabular}{|c|c|c|c|c|c|c|c|}
\hline \multirow[t]{2}{*}{ K-mer } & \multicolumn{3}{|c|}{ Skip-mer } & \multirow[t]{2}{*}{ Secondary Structure Fingerprints } & \multicolumn{3}{|c|}{ Test Set Accuracy } \\
\hline & Length & Match & Skip & & 3-Layers Model & 4-Layers Model & 5-Layers Model \\
\hline 4-mer & & - & & - & $52.72 \%$ & $53.67 \%$ & $59.74 \%$ \\
\hline 5-mer & & - & & - & $72.52 \%$ & $70.29 \%$ & $70.61 \%$ \\
\hline 6-mer & & - & & - & $80.51 \%$ & $75.08 \%$ & $71.57 \%$ \\
\hline- & 6 & 2 & 1 & - & $56.23 \%$ & $62.3 \%$ & $65.81 \%$ \\
\hline- & 7 & 1 & 1 & - & $41.21 \%$ & $44.09 \%$ & $52.08 \%$ \\
\hline- & 7 & 2 & 1 & - & $75.4 \%$ & $71.25 \%$ & $71.88 \%$ \\
\hline- & 9 & 1 & 1 & - & $48.24 \%$ & $64.54 \%$ & $61.98 \%$ \\
\hline- & 9 & 2 & 1 & - & $79.87 \%$ & $71.25 \%$ & $67.73 \%$ \\
\hline- & 11 & 1 & 1 & - & $68.37 \%$ & $68.37 \%$ & $66.45 \%$ \\
\hline- & & - & & $\min$. free energy & $39.94 \%$ & $38.02 \%$ & $39.94 \%$ \\
\hline- & & - & & min., avg. free energy & $51.44 \%$ & $48.88 \%$ & $59.11 \%$ \\
\hline- & & - & & min., avg., max. free energy & $46.33 \%$ & $48.56 \%$ & $48.88 \%$ \\
\hline 4-mer & 6 & 2 & 1 & - & $71.25 \%$ & $70.93 \%$ & $52.72 \%$ \\
\hline 4-mer & 7 & 1 & 1 & - & $72.2 \%$ & $69.33 \%$ & $71.57 \%$ \\
\hline 5-mer & 7 & 2 & 1 & - & $78.27 \%$ & $77.0 \%$ & $73.8 \%$ \\
\hline 5-mer & 9 & 1 & 1 & - & $69.97 \%$ & $65.81 \%$ & $69.65 \%$ \\
\hline 6-mer & 9 & 2 & 1 & - & $76.36 \%$ & $71.88 \%$ & $68.69 \%$ \\
\hline 6-mer & 11 & 1 & 1 & - & $72.84 \%$ & $71.57 \%$ & $53.99 \%$ \\
\hline 4 -mer & & - & & min. free energy & $61.66 \%$ & $61.98 \%$ & $49.2 \%$ \\
\hline 5-mer & & - & & $\min$. free energy & $67.09 \%$ & $72.2 \%$ & $69.01 \%$ \\
\hline 6-mer & & - & & $\min$. free energy & $72.2 \%$ & $69.97 \%$ & $61.98 \%$ \\
\hline 4-mer & & - & & min., avg. free energy & $63.58 \%$ & $59.42 \%$ & $63.9 \%$ \\
\hline 5-mer & & - & & min., avg. free energy & $64.22 \%$ & $66.77 \%$ & $66.77 \%$ \\
\hline 6-mer & & - & & min., avg. free energy & $69.65 \%$ & $63.9 \%$ & $58.47 \%$ \\
\hline 4-mer & & - & & min., avg., max. free energy & $59.74 \%$ & $60.06 \%$ & $65.18 \%$ \\
\hline 5-mer & & - & & min., avg., max. free energy & $65.81 \%$ & $64.22 \%$ & $56.23 \%$ \\
\hline 6-mer & & - & & min., avg., max. free energy & $64.86 \%$ & $63.26 \%$ & $52.08 \%$ \\
\hline- & 6 & 2 & 1 & min. free energy & $60.7 \%$ & $59.42 \%$ & $65.5 \%$ \\
\hline- & 7 & 1 & 1 & $\min$. free energy & $42.17 \%$ & $48.24 \%$ & $47.92 \%$ \\
\hline- & 7 & 2 & 1 & $\min$. free energy & $60.06 \%$ & $69.01 \%$ & $70.29 \%$ \\
\hline- & 9 & 1 & 1 & $\min$. free energy & $44.73 \%$ & $47.6 \%$ & $63.26 \%$ \\
\hline- & 9 & 2 & 1 & $\min$. free energy & $68.69 \%$ & $69.01 \%$ & $68.05 \%$ \\
\hline- & 11 & 1 & 1 & $\min$. free energy & $71.25 \%$ & $68.37 \%$ & $64.86 \%$ \\
\hline- & 6 & 2 & 1 & min., avg. free energy & $53.99 \%$ & $58.47 \%$ & $55.91 \%$ \\
\hline- & 7 & 1 & 1 & min., avg. free energy & $54.31 \%$ & $53.04 \%$ & $52.72 \%$ \\
\hline- & 7 & 2 & 1 & min., avg. free energy & $59.11 \%$ & $67.41 \%$ & $69.33 \%$ \\
\hline- & 9 & 1 & 1 & min., avg. free energy & $63.26 \%$ & $61.66 \%$ & $64.86 \%$ \\
\hline- & 9 & 2 & 1 & min., avg. free energy & $71.57 \%$ & $63.58 \%$ & $65.81 \%$ \\
\hline- & 11 & 1 & 1 & min., avg. free energy & $69.33 \%$ & $66.13 \%$ & $62.62 \%$ \\
\hline- & 6 & 2 & 1 & min., avg., max. free energy & $57.51 \%$ & $57.19 \%$ & $59.74 \%$ \\
\hline- & 7 & 1 & 1 & min., avg., max. free energy & $53.35 \%$ & $53.35 \%$ & $52.08 \%$ \\
\hline- & 7 & 2 & 1 & min., avg., max. free energy & $57.83 \%$ & $62.62 \%$ & $59.74 \%$ \\
\hline- & 9 & 1 & 1 & min., avg., max. free energy & $51.76 \%$ & $63.26 \%$ & $65.81 \%$ \\
\hline- & 9 & 2 & 1 & min., avg., max. free energy & $67.41 \%$ & $68.37 \%$ & $64.22 \%$ \\
\hline- & 11 & 1 & 1 & min., avg., max. free energy & $62.3 \%$ & $63.9 \%$ & $61.66 \%$ \\
\hline 4-mer & 6 & 2 & 1 & min. free energy & $67.09 \%$ & $64.22 \%$ & $65.5 \%$ \\
\hline 4 -mer & 7 & 1 & 1 & $\min$. free energy & $60.7 \%$ & $59.74 \%$ & $47.28 \%$ \\
\hline 5-mer & 7 & 2 & 1 & min. free energy & $77.0 \%$ & $72.2 \%$ & $66.13 \%$ \\
\hline 5-mer & 9 & 1 & 1 & $\min$. free energy & $70.29 \%$ & $72.84 \%$ & $71.88 \%$ \\
\hline 6-mer & 9 & 2 & 1 & $\min$. free energy & $76.04 \%$ & $68.69 \%$ & $66.45 \%$ \\
\hline 6-mer & 11 & 1 & 1 & $\min$. free energy & $78.59 \%$ & $77.32 \%$ & $61.66 \%$ \\
\hline 4 -mer & 6 & 2 & 1 & min., avg. free energy & $60.06 \%$ & $60.38 \%$ & $61.02 \%$ \\
\hline 4-mer & 7 & 1 & 1 & min., avg. free energy & $66.77 \%$ & $66.45 \%$ & $65.18 \%$ \\
\hline 5-mer & 7 & 2 & 1 & min., avg. free energy & $67.73 \%$ & $68.37 \%$ & $63.58 \%$ \\
\hline 5-mer & 9 & 1 & 1 & min., avg. free energy & $70.61 \%$ & $66.77 \%$ & $69.33 \%$ \\
\hline 6-mer & 9 & 2 & 1 & min., avg. free energy & $77.32 \%$ & $69.97 \%$ & $63.58 \%$ \\
\hline 6-mer & 11 & 1 & 1 & min., avg. free energy & $73.48 \%$ & $69.65 \%$ & $57.51 \%$ \\
\hline 4-mer & 6 & 2 & 1 & min., avg., max. free energy & $67.73 \%$ & $65.18 \%$ & $68.69 \%$ \\
\hline 4-mer & 7 & 1 & 1 & min., avg., max. free energy & $59.11 \%$ & $63.9 \%$ & $63.9 \%$ \\
\hline 5-mer & 7 & 2 & 1 & min., avg., max. free energy & $66.13 \%$ & $61.34 \%$ & $66.13 \%$ \\
\hline 5-mer & 9 & 1 & 1 & min., avg., max. free energy & $71.88 \%$ & $68.37 \%$ & $66.13 \%$ \\
\hline 6-mer & 9 & 2 & 1 & min., avg., max. free energy & $77.32 \%$ & $74.76 \%$ & $59.42 \%$ \\
\hline 6-mer & 11 & 1 & 1 & min., avg., max. free energy & $67.09 \%$ & $62.3 \%$ & $58.15 \%$ \\
\hline
\end{tabular}


we did not limit our dataset to specific species of RNA viruses. A 10 -fold cross-validated highest prediction accuracy of $86.89 \%$ was achieved by combining 6-mer with match-2-skip-1 "skip-mers" [6] of length 9 . However, preliminary evaluation of the feature sets with artificially limited datasets suggests that this combination may not necessarily be the best performing set for a more complete dataset in the future, as the best performing set of features was found to be different for each of the datasets. In addition, our results indicate that the structure-based features failed to provide a significant advantage to the overall performance of the trained deep neural networks, despite the conservation $[17,41]$ and known function of RNA secondary structures in certain virus-host interactions [18].

When it comes to limitation, our approach has only been evaluated with datasets from a single source, the NCBI Virus [3]. This means that any possible limitations in the source, such as possibly missing hosts for a sequence of RNA virus, could have impacted the evaluation of our proposed approach. Furthermore, for a single sequence of RNA virus, only the top host predicted by the neural network is currently considered - this study has not investigated the lower-ranked predicted hosts, which may or may not be susceptible or act as reservoir hosts. Finally, the approach has only been developed for and tested with complete sequences of RNA viruses, whereas the majority of RNA virus sequences available from NCBI Virus are annotated as "partial" [3]. A future study could adapt our approach so that host susceptibility can be predicted from partial viral sequences, which may be useful for cases where only incomplete sequences of a specific virus are available.

\section{AVAILABILITY}

The dataset used in this study (with the secondary structure fingerprints) is available at: https://www.eecs.uottawa.ca/ turcotte/ icbbt2021.

\section{ACKNOWLEDGMENTS}

This study was supported by funding from Natural Sciences and Engineering Research Council of Canada, and computing facilities provided by Compute Ontario and Compute Canada.

\section{REFERENCES}

[1] Martín Abadi, Ashish Agarwal, Paul Barham, Eugene Brevdo, Zhifeng Chen, Craig Citro, Greg S. Corrado, Andy Davis, Jeffrey Dean, Matthieu Devin, Sanjay Ghemawat, Ian Goodfellow, Andrew Harp, Geoffrey Irving, Michael Isard, Yangqing Jia, Rafal Jozefowicz, Lukasz Kaiser, Manjunath Kudlur, Josh Levenberg, Dandelion Mané, Rajat Monga, Sherry Moore, Derek Murray, Chris Olah, Mike Schuster, Jonathon Shlens, Benoit Steiner, Ilya Sutskever, Kunal Talwar, Paul Tucker, Vincent Vanhoucke, Vijay Vasudevan, Fernanda Viégas, Oriol Vinyals, Pete Warden, Martin Wattenberg, Martin Wicke, Yuan Yu, and Xiaoqiang Zheng. 2015. TensorFlow: Large-Scale Machine Learning on Heterogeneous Systems. https://www.tensorflow.org/ Software available from tensorflow.org.

[2] Nathan A Ahlgren, Jie Ren, Yang Young Lu, Jed A Fuhrman, and Fengzhu Sun 2016. Alignment-free $d_{2}^{*}$ oligonucleotide frequency dissimilarity measure improves prediction of hosts from metagenomically-derived viral sequences. $\mathrm{Nu}$ cleic Acids Research 45, 1 (11 2016), 39-53. https://doi.org/10.1093/nar/gkw1002 arXiv:https://academic.oup.com/nar/article-pdf/45/1/39/29193437/gkw1002.pdf

[3] J. Rodney Brister, Danso Ako-adjei, Yiming Bao, and Olga Blinkova. 2015. NCBI Viral Genomes Resource. Nucleic Acids Research 43, D1 (Jan. 2015), D571-D577. https://doi.org/10.1093/nar/gku1207

[4] Stefan Burkhardt and Juha Kärkkäinen. 2003. Better Filtering with Gapped q-Grams. Fundamenta Informaticae 56, 1-2 (2003), 51-70. Publisher: IOS Press.

[5] François Chollet et al. 2015. Keras. https://keras.io.

[6] Bernardo J. Clavijo, Gonzalo Garcia Accinelli, Luis Yanes, Katie Barr, and Jonathan Wright. 2017. Skip-mers: increasing entropy and sensitivity to detect conserved genic regions with simple cyclic q-grams. bioRxiv (2017). https://doi.org/10. $1101 / 179960$

[7] S. Cleaveland, M.K. Laurenson, and L.H. Taylor. 2001. Diseases of humans and their domestic mammals: pathogen characteristics, host range and the risk of emergence. Philosophical Transactions of the Royal Society of London. Series B: Biological Sciences 356, 1411 (July 2001), 991-999. https://doi.org/10.1098/rstb. 2001.0889

[8] T. Jonathan Davies and Amy B Pedersen. 2008. Phylogeny and geography predict pathogen community similarity in wild primates and humans. Proceedings of the Royal Society B: Biological Sciences 275, 1643 (July 2008), 1695-1701. https: //doi.org/10.1098/rspb.2008.0284

[9] Christine LP Eng, Joo Chuan Tong, and Tin Wee Tan. 2014. Predicting host tropism of influenza A virus proteins using random forest. BMC Medical Genomics 7, 3 (Dec. 2014), S1. https://doi.org/10.1186/1755-8794-7-S3-S1

[10] Martin T. Ferris, Paul Joyce, and Christina L. Burch. 2007. High Frequency of Mutations That Expand the Host Range of an RNA Virus. Genetics 176, 2 (June 2007), 1013-1022. https://doi.org/10.1534/genetics.106.064634

[11] Dale Fisher and David Heymann. 2020. Q\&A: The novel coronavirus outbreak causing COVID-19. BMC Medicine 18, 1 (Dec. 2020), 57. https://doi.org/10.1186/ s12916-020-01533-w

[12] Limin Fu, Beifang Niu, Zhengwei Zhu, Sitao Wu, and Weizhong Li. 2012. CD-HIT: accelerated for clustering the next-generation sequencing data. Bioinformatics 28, 23 (Dec. 2012), 3150-3152. https://doi.org/10.1093/bioinformatics/bts565

[13] Tadayoshi Fushiki. 2011. Estimation of prediction error by using K-fold crossvalidation. Statistics and Computing 21, 2 (April 2011), 137-146. https://doi.org/ 10.1007/s11222-009-9153-8

[14] Clovis Galiez, Matthias Siebert, François Enault, Jonathan Vincent, and Johannes Söding. 2017. WIsH: who is the host? Predicting prokaryotic hosts from metagenomic phage contigs. Bioinformatics 33, 19 (07 2017), 3113-3114. https://doi.org/10.1093/ bioinformatics/btx383 arXiv:https://academic.oup.com/bioinformatics/articlepdf $/ 33 / 19 / 3113 / 25164881 /$ btx383.pdf

[15] Feng Gao, Elizabeth Bailes, David L. Robertson, Yalu Chen, Cynthia M. Rodenburg, Scott F. Michael, Larry B. Cummins, Larry O. Arthur, Martine Peeters, George M. Shaw, Paul M. Sharp, and Beatrice H. Hahn. 1999. Origin of HIV-1 in the chimpanzee Pan troglodytes troglodytes. Nature 397, 6718 (Feb. 1999), 436-441. https://doi.org/10.1038/17130

[16] Masami Hasegawa and Takashi Miyata. 1980. On the antisymmetry of the amino acid code table. Origins of life 10, 3 (1980), 265-270.

[17] Ivo L Hofacker and Peter F Stadler. 1999. Automatic detection of conserved base pairing patterns in RNA virus genomes. Computers \& Chemistry 23, 3-4 (June 1999), 401-414. https://doi.org/10.1016/S0097-8485(99)00013-3

[18] J. L. Hyde, C. L. Gardner, T. Kimura, J. P. White, G. Liu, D. W. Trobaugh, C. Huang, M. Tonelli, S. Paessler, K. Takeda, W. B. Klimstra, G. K. Amarasinghe, and M. S. Diamond. 2014. A Viral RNA Structural Element Alters Host Recognition of Nonself RNA. Science 343, 6172 (Feb. 2014), 783-787. https://doi.org/10.1126/ science. 1248465

[19] Diederik P. Kingma and Jimmy Ba. 2017. Adam: A Method for Stochastic Optimization. arXiv:1412.6980 [cs.LG]

[20] Jessime M. Kirk, Susan O. Kim, Kaoru Inoue, Matthew J. Smola, David M. Lee, Megan D. Schertzer, Joshua S. Wooten, Allison R. Baker, Daniel Sprague, David W. Collins, Christopher R. Horning, Shuo Wang, Qidi Chen, Kevin M. Weeks, Peter J. Mucha, and J. Mauro Calabrese. 2018. Functional classification of long noncoding RNAs by k-mer content. Nature Genetics 50, 10 (Oct. 2018), 1474-1482. https://doi.org/10.1038/s41588-018-0207-8

[21] Han Li and Fengzhu Sun. 2018. Comparative studies of alignment, alignment-free and SVM based approaches for predicting the hosts of viruses based on viral sequences. Scientific Reports 8, 1 (July 2018), 10032. https://doi.org/10.1038/ s41598-018-28308-x

[22] W. Li and A. Godzik. 2006. Cd-hit: a fast program for clustering and comparing large sets of protein or nucleotide sequences. Bioinformatics 22, 13 (July 2006), 1658-1659. https://doi.org/10.1093/bioinformatics/btl158

[23] Yu Li, Chao Huang, Lizhong Ding, Zhongxiao Li, Yijie Pan, and Xin Gao. 2019. Deep learning in bioinformatics: Introduction, application, and perspective in the big data era. Methods 166 (Aug. 2019), 4-21. https://doi.org/10.1016/j.ymeth. 2019.04.008

[24] Bin Liu, Longyun Fang, Shanyi Wang, Xiaolong Wang, Hongtao Li, and KuoChen Chou. 2015. Identification of microRNA precursor with the degenerate K-tuple or Kmer strategy. Journal of Theoretical Biology 385 (Nov. 2015), 153-159. https://doi.org/10.1016/j.jtbi.2015.08.025

[25] Ping Liu, Jing-Zhe Jiang, Xiu-Feng Wan, Yan Hua, Linmiao Li, Jiabin Zhou, Xiaohu Wang, Fanghui Hou, Jing Chen, Jiejian Zou, and Jinping Chen. 2020. Are pangolins the intermediate host of the 2019 novel coronavirus (SARS-CoV-2)? PLOS Pathogens 16, 5 (May 2020), e1008421. https://doi.org/10.1371/journal.ppat. 1008421

[26] Ben Longdon, Michael A. Brockhurst, Colin A. Russell, John J. Welch, and Francis M. Jiggins. 2014. The Evolution and Genetics of Virus Host Shifts. PLoS Pathogens 10, 11 (Nov. 2014), e1004395. https://doi.org/10.1371/journal.ppat. 1004395 
[27] T. J. Macke. 2001. RNAMotif, an RNA secondary structure definition and search algorithm. Nucleic Acids Research 29, 22 (Nov. 2001), 4724-4735. https://doi.org/ 10.1093/nar/29.22.4724

[28] John S Mackenzie and Martyn Jeggo. 2013. Reservoirs and vectors of emerging viruses. Current Opinion in Virology 3, 2 (April 2013), 170-179. https://doi.org/ 10.1016/j.coviro.2013.02.002

[29] Ilaria Manfredonia, Chandran Nithin, Almudena Ponce-Salvatierra, Pritha Ghosh Tomasz K Wirecki, Tycho Marinus, Natacha S Ogando, Eric J Snijder, Martijn J van Hemert, Janusz M Bujnicki, et al. 2020. Genome-wide mapping of SARSCoV-2 RNA structures identifies therapeutically-relevant elements. Nucleic acids research (2020).

[30] José Millán-Oñate, Alfonso J. Rodriguez-Morales, German Camacho-Moreno, Henry Mendoza-Ramírez, Iván Arturo Rodríguez-Sabogal, and Carlos ÁlvarezMoreno. 2020. A new emerging zoonotic virus of concern: the 2019 novel Coronavirus (COVID-19). Infectio 24, 3 (April 2020), 187. https://doi.org/10.22354/in. v24i3.848

[31] Florian Mock, Adrian Viehweger, Emanuel Barth, and Manja Marz. 2020. VIDHOP, viral host prediction with Deep Learning. Bioinformatics $(08$ 2020). https://doi.org/10.1093/bioinformatics/btaa705 arXiv:https://academic.oup.com/bioinformatics/advance-articlepdf/doi/10.1093/bioinformatics/btaa705/33637195/btaa705.pdf btaa705.

[32] Nardus Mollentze and Daniel G. Streicker. 2020. Viral zoonotic risk is homogenous among taxonomic orders of mammalian and avian reservoir hosts. Proceedings of the National Academy of Sciences 117, 17 (April 2020), 9423-9430. https: //doi.org/10.1073/pnas.1919176117

[33] David R. Morris and Adam P. Geballe. 2000. Upstream Open Reading Frames as Regulators of mRNA Translation. Molecular and Cellular Biology 20, 23 (Dec. 2000), 8635-8642. https://doi.org/10.1128/MCB.20.23.8635-8642.2000

[34] Vinod Nair and Geoffrey E. Hinton. 2010. Rectified Linear Units Improve Restricted Boltzmann Machines. In ICML. 807-814. https://icml.cc/Conferences/ 2010/papers/432.pdf

[35] Kevin J. Olival, Parviez R. Hosseini, Carlos Zambrana-Torrelio, Noam Ross, Tiffany L. Bogich, and Peter Daszak. 2017. Host and viral traits predict zoonotic spillover from mammals. Nature 546, 7660 (June 2017), 646-650. https://doi.org/10.1038/nature22975

[36] Xiaoyong Pan and Hong-Bin Shen. 2018. Predicting RNA-protein binding sites and motifs through combining local and global deep convolutional neural networks. Bioinformatics 34, 20 (Oct. 2018), 3427-3436. https://doi.org/10.1093/ bioinformatics/bty364

[37] Ramya Rangan, Ivan N. Zheludev, Rachel J. Hagey, Edward A. Pham, Hannah K. Wayment-Steele, Jeffrey S. Glenn, and Rhiju Das. 2020. RNA genome conservation and secondary structure in SARS-CoV-2 and SARS-related viruses: a first look. RNA 26, 8 (Aug. 2020), 937-959. https://doi.org/10.1261/rna.076141.120

[38] Jie Ren, Nathan A. Ahlgren, Yang Young Lu, Jed A. Fuhrman, and Fengzhu Sun. 2017. VirFinder: a novel k-mer based tool for identifying viral sequences from assembled metagenomic data. Microbiome 5, 1 (Dec. 2017), 69. https: //doi.org/10.1186/s40168-017-0283-5

[39] Jie Ren, Kai Song, Chao Deng, Nathan A. Ahlgren, Jed A. Fuhrman, Yi Li, Xiaohui Xie, Ryan Poplin, and Fengzhu Sun. 2020. Identifying viruses from metagenomic data using deep learning. Quantitative Biology 8, 1 (March 2020), 64-77. https: //doi.org/10.1007/s40484-019-0187-4

[40] Jürgen Schmidhuber. 2015. Deep learning in neural networks: An overview. Neural Networks 61 (Jan. 2015), 85-117. https://doi.org/10.1016/j.neunet.2014.09. 003

[41] P. Simmonds and D. B. Smith. 1999. Structural Constraints on RNA Virus Evolution. Fournal of Virology 73, 7 (July 1999), 5787-5794. https://doi.org/10.1128/ JVI.73.7.5787-5794.1999

[42] Kevin Sutanto and Marcel Turcotte. in press. Assessing the Use of Secondary Structure Fingerprints and Deep Learning to Classify RNA Sequences. In IEEE International Conference on Bioinformatics and Biomedicine (BIBM), December 16-19, 2020. Seoul, South Korea.

[43] Louise H. Taylor, Sophia M. Latham, and Mark E.J. Woolhouse. 2001. Risk factors for human disease emergence. Philosophical Transactions of the Royal Society of London. Series B: Biological Sciences 356, 1411 (July 2001), 983-989. https://doi.org/10.1098/rstb.2001.0888

[44] Dhanasekaran Vijaykrishna, Reshmi Mukerji, and Gavin J. D. Smith. 2015. RNA Virus Reassortment: An Evolutionary Mechanism for Host Jumps and Immune Evasion. PLOS Pathogens 11, 7 (July 2015), e1004902. https://doi.org/10.1371/ journal.ppat.1004902

[45] Mark Woolhouse and Eleanor Gaunt. 2007. Ecological Origins of Novel Human Pathogens. Critical Reviews in Microbiology 33, 4 (Jan. 2007), 231-242. https //doi.org/10.1080/10408410701647560

[46] Mark E.J. Woolhouse, Daniel T. Haydon, and Rustom Antia. 2005. Emerging pathogens: the epidemiology and evolution of species jumps. Trends in Ecology \& Evolution 20, 5 (May 2005), 238-244. https://doi.org/10.1016/j.tree.2005.02.009
[47] Cheng-Yan Wu, Qian-Zhong Li, and Zhen-Xing Feng. 2016. Non-coding RNA identification based on topology secondary structure and reading frame in organelle genome level. Genomics 107, 1 (Jan. 2016), 9-15. https://doi.org/10.1016/ j.ygeno.2015.12.002

[48] Mengge Zhang, Lianping Yang, Jie Ren, Nathan A. Ahlgren, Jed A. Fuhrman, and Fengzhu Sun. 2017. Prediction of virus-host infectious association by supervised learning methods. BMC Bioinformatics 18, 3 (March 2017), 60. https://doi.org/10. 1186/s12859-017-1473-7

[49] Tao Zhang, Qunfu Wu, and Zhigang Zhang. 2020. Probable Pangolin Origin of SARS-CoV-2 Associated with the COVID-19 Outbreak. Current Biology 30, 7 (April 2020), 1346-1351.e2. https://doi.org/10.1016/j.cub.2020.03.022

[50] Nantao Zheng, Kairou Wang, Weihua Zhan, and Lei Deng. 2019. Targeting Virushost Protein Interactions: Feature Extraction and Machine Learning Approaches. Current Drug Metabolism 20, 3 (May 2019), 177-184. https://doi.org/10.2174/ 1389200219666180829121038 